\title{
A formal ideal-based account of typicality
}

\author{
Wouter Voorspoels • Wolf Vanpaemel • Gert Storms
}

Published online: 29 June 2011

(C) Psychonomic Society, Inc. 2011

\begin{abstract}
Inspired by Barsalou's (Journal of Experimental Psychology: Learning, Memory, and Cognition, 11, 629$654,1985)$ proposal that categories can be represented by ideals, we develop and test a computational model, the ideal dimension model (IDM). The IDM is tested in its account of the typicality gradient for 11 superordinate natural language concepts and, using Bayesian model evaluation, contrasted with a standard exemplar model and a central prototype model. The IDM is found to capture typicality better than do the exemplar model and the central tendency prototype model, in terms of both goodness of fit and generalizability. The present findings challenge the dominant view that exemplar representations are most successful and present compelling evidence that superordinate natural language categories can be represented using an abstract summary, in the form of ideal representations. Supplemental appendices for this article can be downloaded from http://mc. psychonomic-journals.org/content/supplemental.
\end{abstract}

Keywords Typicality . Computational model .

Representation · Bayesian model evaluation · Exemplar.

Prototype $\cdot$ Ideal

Electronic supplementary material The online version of this article (doi:10.3758/s13423-011-0122-9) contains supplementary material, which is available to authorized users.

W. Voorspoels $\cdot$ W. Vanpaemel · G. Storms

University of Leuven,

Leuven, Belgium

W. Voorspoels $(\bowtie)$

Department of Psychology, University of Leuven,

Tiensestraat 102,

3000 Leuven, Belgium

e-mail: wouter.voorspoels@psy.kuleuven.be
In a classic contribution to category representation research, Barsalou (1985) proposed the notion of ideals. He observed that, as in common natural language categories, in goalderived categories (e.g., things you eat when on a diet), some members are generally considered better examples of the category than are other members. However, whereas, in common natural language categories, the graded internal structure depends largely on similarity to the prototype of the category, Barsalou provided compelling evidence that, for goal-derived categories, the typicality gradient is largely determined by an extreme, ideal value on a relevant dimension. For example, whereas a blackbird is a typical bird because it is similar to the average bird, porridge is considered a typical member of the goal-derived category things you eat when on a diet, not because it is similar to the average diet product, but because it is low on calories; that is, it is close to the ideal value on that dimension (zero calories). Clearly, the average diet product does not contain zero calories.

The notion of ideals in category representation has since then been influential in research regarding category learning and representation (e.g., Atran, 1999; Burnett, Medin, Ross, \& Blok, 2005; Davis \& Love, 2010; Lynch, Coley, \& Medin, 2000). Yet it has never arrived at the level of a representational, computational model. In the present study, we propose and test such a computational model of ideal representation.

\section{Three views on typicality}

The prototype view (e.g., Hampton, 1979; Posner \& Keele, 1968; Rosch, 1975; Younger \& Cohen, 1983) states that a category is represented by an abstract summary, referred to as a prototype. In this view, the concept vehicle is a 
summary representation of what vehicles are generally like, abstracted from specific instances of vehicles, containing information such as moves people or cargo from point $A$ to point B. Car is a typical member of the category vehicle because it is highly similar to the abstract vehicle prototype, while hot air balloon is atypical since it is very dissimilar to the abstract summary. In general, the prototype is conceived of as the central tendency, the average, of the category.

The exemplar view (Brooks, 1978; Medin \& Schaffer, 1978), on the other hand, proposes that a category is represented by previously encountered instances of the category, and typicality is conceptualized as the summed similarity of a category member to all the stored members of the category. While it is debatable what an exemplar is in the context of natural language concepts (Heit \& Barsalou, 1996), it is often practically assumed that exemplars are subordinate categories one level lower than the activated concept (e.g., Komatsu, 1992). In this view, the concept vehicle consists of representations of previously encountered instances of vehicles, such as train, plane, and metro. Car is a typical vehicle because it is highly similar to many stored instances of vehicle, while hot air balloon is atypical, since it is similar to few stored instances.

Barsalou's (1985) notion of ideal representation presents an intriguing alternative to the traditional prototype and exemplar approaches. In this view, a category is represented by an ideal, constituted by the ideal dimension: a relevant characteristic or a combination of ideal features. In terms of typicality, the more a category member has of whatever it is the ideal dimension of the category consists of, the more typical the member is of that category. More is better; that is, extreme, rather than average, values on a relevant dimension are important: The lower a diet product is on calories, the more it is typical of the category things you eat when on a diet, and the more valuable something is, the more typical it is of things you rescue from a burning house.

Barsalou (1985) originally launched the notion of ideal representation in goal-derived categories, where the ideal dimension is closely linked to a certain goal (e.g., calories in the category things to eat when on a diet). The potential scope of ideal representation should not be restricted to this type of category. In fact, it has been found that for tree experts, height and weediness are ideal dimensions for tree typicality (taller and less weedy trees are rated as more typical; Lynch et al., 2000); for fishermen, culturally determined desirability is an ideal dimension for fish typicality (Burnett et al., 2005); and for the Itzai Maya from Guatemala, beauty and meat tastiness are ideal dimensions for bird typicality (Atran, 1999). In these examples, a direct link to an explicit goal is less obvious. Interestingly, Barsalou observed some influence of ideal representations in common natural language concepts, even when no goal whatsoever was defined. In sum, these findings suggest that the notion of ideals can be extended to other types of categories, such as natural language concepts.

\section{Overview}

The prototype and exemplar views have been rigorously formalized as computational models, allowing a precise and quantitative test of the models. The most influential model implementing the exemplar view is the generalized context model (GCM) of category learning (Nosofsky, 1986), later successfully adapted to account for typicality judgments as well (e.g., Nosofsky, 1988). An often-tested prototype model is the central prototype model ( CPM; e.g., Minda \& Smith, 2010; Nosofsky, 1992), in which the prototype of a category is assumed to be the average member, retrieved in a spatial similarity representation by averaging across the coordinates of all members on each dimension. In general, both in category-learning experiments and in natural language concept research, when the prototype and exemplar models are compared, exemplar models fare better as models of categorization and typicality (e.g., Nosofsky, 1992; Smits, Storms, Rosseel, \& De Boeck, 2002).

The ideal representation view, in contrast, has escaped careful computational implementation. This lack of formal implementation has implied that the ideal view has never gained the same attention and authority as the prototype and exemplar views. To fill the void, this article proposes a computational model, referred to as the ideal dimension model (IDM), which implements ideal representations in geometric similarity spaces. After describing the model, we discuss the data used to test the models, involving 11 superordinate concepts from the animal and artifact domains. Then, the GCM, CPM, and the newly proposed IDM are tested on how well they can account for the typicality gradient of natural language concepts. We end with a discussion of the interpretation of the ideal dimension and the relevance of our findings for the debate on abstraction in representation.

\section{The IDM}

The IDM assumes that a single ideal dimension exists that captures the typicality structure in a category. The typicality of an exemplar corresponds to the value of the exemplar on that specific ideal dimension. The more extreme this value is located along the dimension in the ideal direction, the more typical the exemplar is. The left panel of Fig. 1 provides a graphical illustration of the IDM. A set of eight mammals is depicted by circles in a hypothetical two- 
Fig. 1 Graphical representation of typicality according to the IDM. The left panel presents a (fictive) representation of a set of members of the category mammals. The solid line with the arrow represents the ideal dimension that determines the typicality of the members for the category according to the IDM. The members are orthogonally projected onto the ideal dimension, and these projections can be considered dimensional values along the ideal dimension. These values are the modelbased typicality scores of the members for the category, as presented in the right panel
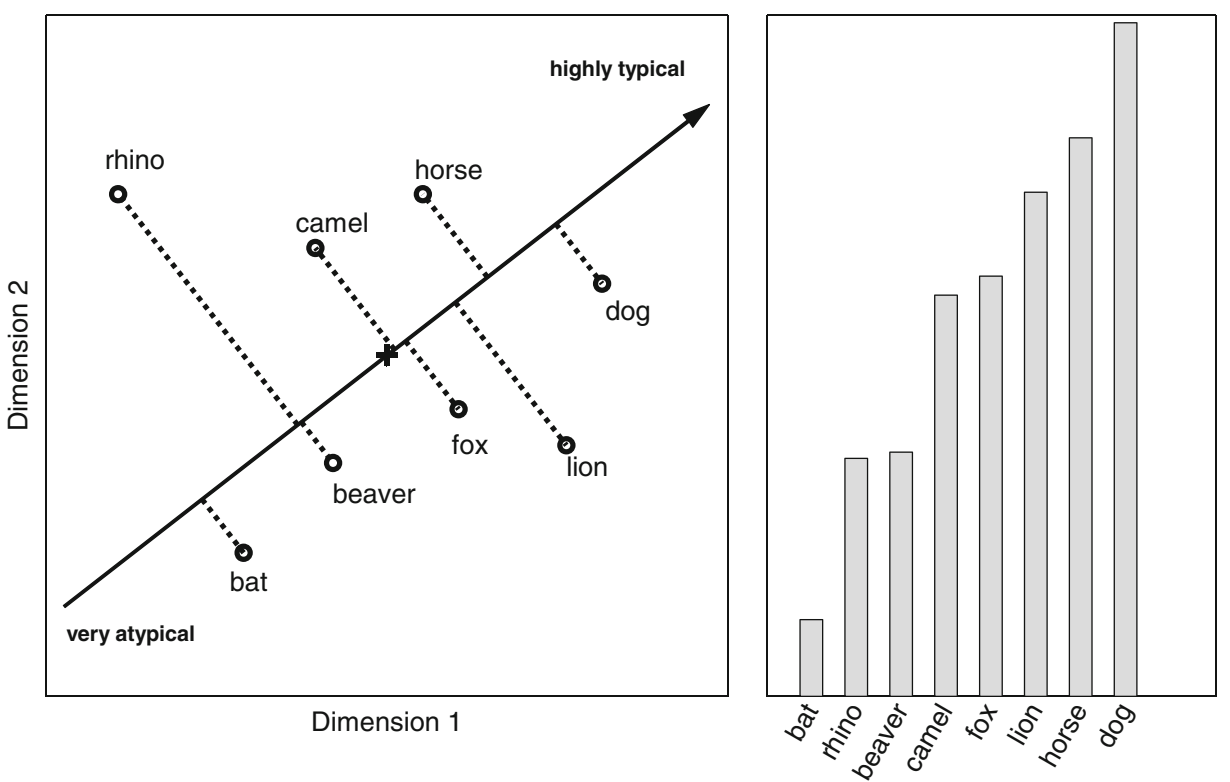

dimensional spatial representation. The diagonal straight line with the arrow represents the ideal dimension of the category mammals, and typicality for mammals is determined by the orthogonal projection of exemplars along that dimension. The right panel presents the typicality predictions of the IDM for the members. It can be seen that projections on the ideal dimension that are further in the ideal direction lead to a higher predicted typicality. In other words, the more a certain mammal has of whatever constitutes the ideal dimension, the more typical it is for mammals.

Formally, the IDM assumes that judging the typicality of an exemplar $i$ for a category $A$ comes down to evaluating the value of $i$ on a single dimension $V_{A}$, determined by the origin and point $a$. In an $M$-dimensional space, the typicality of exemplar $i$ for category $A$, is then given by:

$t_{i A}=\frac{\sum_{k=1}^{M} x_{i k} x_{a k}}{\left(\sum_{k=1}^{M}\left(x_{a k}\right)^{2}\right)^{1 / 2}}$

where $x_{a k}$ are the coordinates of a point $a$ that, together with the origin, determines the ideal dimension $V_{A}$ and $x_{i k}$ are the coordinates of exemplar $i$. The free parameters in the model are the coordinates of point $a$. The distance from the origin to point $a,|o a|$, can be arbitrarily fixed.

In Eq. 1, the point representing exemplar $i$ in the representational space is projected orthogonally onto the dimension $V_{A}$. This dimension can be considered a feature (or combination of features) that is relevant in the similarity structure of the category. On the basis of the orthogonal projection of exemplar $i$, a dimensional value along $V_{A}$ is derived, measured in units of length $|o a|$ relative to the origin (point zero). The dimensional value of exemplar $i$ is its typicality for the category. The further the projection of an exemplar is in the ideal direction, as determined by $V_{A}$, the higher the predicted typicality is. In Fig. 1, dog is predicted to be the most typical member in the set, since its projection on the ideal dimension is furthest in the ideal direction. Rhino and beaver are predicted to be rather atypical and nearly identical in typicality, following from their projection on the ideal dimension.

In Fig. 1, the ideal dimension runs through the origin (the cross) of the space. It can, however, be easily seen that any line parallel to the ideal dimension would produce the same typicality prediction. Lines that are not parallel will yield different predictions. Since in a two-dimensional space, all lines with an identical slope parameter are parallel lines and, thus, yield identical typicality predictions, it follows that knowing the slope parameter allows one to derive the corresponding typicality predictions. Following the same reasoning, in an $M$-dimensional space, the IDM is fully determined by $M-1$ slope parameters. In other words, to define the set of all ideal dimensions that lead to unique typicality predictions, $M-1$ free parameters are required.

Intuitively, one can usefully think of the IDM as predicting typicality on the basis of the distance to an ideal point at infinity on the ideal dimension (see, e.g., Carroll, 1972, in the context of models of preference data). This is illustrated in Fig. 2, which again presents the spatial representation of the mammals and illustrates the distance toward an ideal point, indicated by the star that moves far away on the ideal dimension. Calculating the distance to the ideal prototype results in a prediction of the typicality 


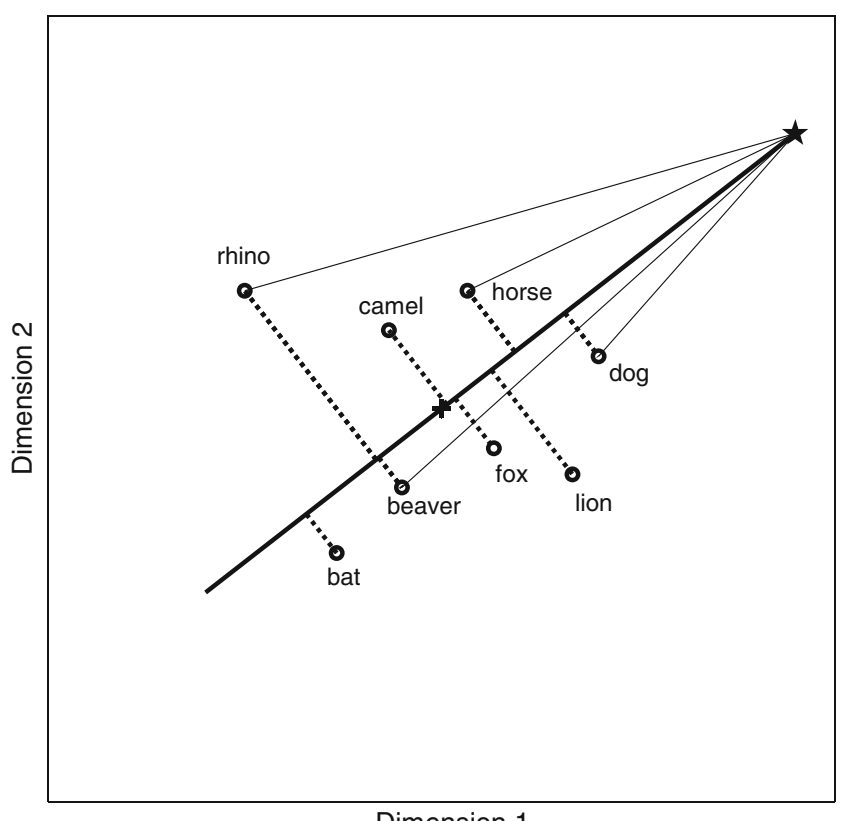

Dimension 1

Fig. 2 Illustration of the equivalence of the IDM and an ideal point at infinity. The solid lines reflect the distance toward an ideal point (the star), representing the typicality according to an ideal point model. The typicality prediction of an ideal point approximates the prediction of the IDM more as it is further away

gradient very similar to the orthogonal projection of the exemplars on the ideal dimension. It can be seen in Fig. 2 that the relative differences in typicality between exemplars in terms of the distance to the ideal point are nearly identical to the relative differences between exemplars in terms of orthogonal projection on the ideal dimension. The equivalence holds only when the ideal point is far from the category, reaching perfection when it is at infinity.

In sum, the IDM is an abstractionist model that proposes an abstract summary representation. Contrary to a central tendency prototype, the ideal dimension does not specify what the members of a category are like on average but, rather, contains extreme, ideal information, perhaps stipulating what members of the category should be like.

\section{Data}

The present study focuses on 11 superordinate categories in two semantic domains: animals and artifacts. The data were taken from a recent norm study of De Deyne et al. (2008). The animal domain contains 129 exemplars in five categories: birds, fish, insects, mammals, and reptiles. The artifact domain contains 165 exemplars in six categories: clothing, kitchen utensils, musical instruments, tools, vehicles, and weapons. All categories contain between 20 and 33 exemplars.
Typicality ratings

Typicality was measured by judgments of goodness of example of members for their category. We relied on ratings reported by De Deyne et al. (2008). The exemplars of each category, presented as verbal stimuli, were rated by 28 participants for goodness of example for the superordinate category they belonged to on a Likert-rating scale ranging from 1 for very bad examples to 20 for very good examples. The judgments were averaged across participants. The reliability, evaluated by split-half correlations corrected with the Spearman-Brown formula, ranged from .91 to .98 across the 11 categories (De Deyne et al., 2008, Table 1, p. 1033). The data showed a clear typicality gradient for all categories, with typicality ratings varying, on average, from 8.20 to 18.80 across category members, with a standard deviation of 3.08 .

Derived similarity measure and geometric representation

The IDM is based on underlying spatial representations in which exemplars are represented as points in an $M$ dimensional space. To construct the spatial similarity representations of the two semantic domains, we used a pairwise similarity measure derived from feature data.

De Deyne et al. (2008) created an exemplar $\times$ feature matrix containing all exemplars and all features of a domain. In total, 1,003 participants generated 765 unique features for animals and 1,295 unique features for artifacts, covering a broad range of features. Four participants judged the applicability of each feature for each exemplar. The split-half reliability of these judgments was .83 for the animals matrix and .81 for the artifacts matrix (see De Deyne et al., 2008, p. 1042).

We derived a pairwise similarity measure by summing the feature $\times$ exemplar matrices of each domain across participants and correlating the summed feature vectors of all exemplar pairs within a domain. Dry and Storms (2009) have shown that this procedure provides a good similarity measure.

On the basis of the similarity matrices, we derived spatial representations using an SAS nonmetric MDS

Table 1 Percentages of triangle inequality violations (TIVs) and stress values for the two semantic domains as a function of the dimensionality of the MDS solution

\begin{tabular}{lllllll}
\hline & \multirow{2}{*}{ TIV } & \multicolumn{5}{l}{ Stress Values } \\
\cline { 3 - 7 } & & $2 \mathrm{D}$ & 3D & 4D & $5 \mathrm{D}$ & $6 \mathrm{D}$ \\
\hline Animals & $0.13 \%$ & .18 & .12 & .07 & .06 & .04 \\
Artifacts & $<0.01 \%$ & .16 & .10 & .07 & .06 & .05 \\
\hline
\end{tabular}


analysis (SAS, V9). In the interest of generality, solutions in two to six dimensions were considered. The appropriateness of a spatial representation for the domains was evaluated by calculating the percentage of triplets in the similarity matrices that violate the triangle inequality and a badness-of-fit measure (stress) for the MDS solutions.

As can be seen in Table 1, both the percentages of triangle inequality violations and the stress values suggest the appropriateness of the MDS solutions. Following Kruskal (1964), solutions with stress values exceeding .10 are not considered for further analyses, leaving representations with dimensionality from four to six for further analysis.

\section{Model evaluation and comparison}

To evaluate the IDM as a model of typicality and, by extension, of category representation, we ask two fundamental questions. First, can the model adequately account for the typicality gradient in the 11 natural language concepts? We will do this by evaluating the IDM on its optimal fit. The second question concerns the competitiveness of the IDM with established and more traditional models of category representation. To investigate this question, we will compare the IDM with an exemplar model, the GCM, arguably the most successful categorization model, and with the CPM, a prototype model that assumes a central tendency prototype. Rather than on goodness of fit, model comparison should rely on a measure of generalizability, such as the marginal likelihood (Myung, 2000; Pitt, Kim, \& Myung, 2003). Formal details of the models can be found in the online supplementary Appendix A. Detailed information on the methods of model evaluation is given in the online Appendix B.

\section{Goodness of fit}

We tested whether the models are able to capture the typicality gradient of natural language concepts. For each model, we optimized the correlation between model-based typicality scores (i.e., the model-based typicality prediction for each category exemplar) and observed typicality scores of the category exemplars. This was done for all categories and dimensionalities separately. The optimized correlations are presented in Fig. 3. It is clear that the IDM can be considered an adequate model of typicality: The mean optimal correlation for the animal categories is .88 in representations with six underlying dimensions and .82 in the artifact domain. The GCM achieves a satisfactory correlation of .65 for the animal categories and .76 for the artifact categories (again averaged across categories in dimensionality 6). The CPM seems to have the most trouble accounting for the observations, reaching an average correlation of .64 for the animal categories and .62 for the artifact categories in dimensionality 6 . In short, all models can produce decent optimal correlations with the observed typicality ratings, with the IDM having the advantage over the other models.

Interestingly, these analyses are heavily in favor of the IDM. For all categories, the IDM can provide an optimal account of typicality that is closer to the observations than the central tendency prototype model, and even the exemplar model. The GCM is the better model only for the reptiles category in dimensionality 4 and in the tools category in dimensionalities 4 and 5. The CPM provides the best optimal correlation for none of the 11 categories and performs worse than the GCM for nearly all tested categories and dimensionalities.

Providing a satisfactory optimal fit is a necessary condition for any computational model to be taken seriously. However, given the potential differences in flexibility between models, mere goodness of fit is not an adequate criterion for model comparison. Rather, model comparison should ideally rely on generalizability, which balances goodness of fit with complexity. To this end, we tested the models using marginal likelihood, which assesses the average fit of the model across all possible parameter values, rather than the best fit at the optimally chosen parameter value (Lee, 2004; Lee \& Wagenmakers, 2005; Myung, 2000; Myung \& Pitt, 1997; Vanpaemel \& Storms, 2010). In this way, differences between the models in terms of number of free parameters and functional form of the mathematical expressions are acknowledged and accounted for.

\section{Generalizability}

The results of the analyses using marginal likelihood are reported in Fig. 4 in terms of model weights, reflecting the relative evidence that the data provide in favor of that model, within the set of all models that are evaluated (Burnham \& Anderson, 2002; Lee, 2004; Wagenmakers \& Farrel, 2004). For example, the model weight of the GCM is the marginal likelihood of the GCM divided by the sum of marginal likelihoods of the GCM, CPM, and IDM. The higher the relative marginal likelihood of a particular model, the more it is favored by the data, relative to the other models under consideration.

We relied on standard uninformative priors for calculating the marginal likelihoods. For the IDM, a uniform prior over all points at a given distance of the origin was used. Together with the origin, these points determine all possible ideal dimensions with a unique typicality prediction in the space. For the GCM, a uniform prior over the range 0 to 1 was used for the dimensional weights, adding the restriction 

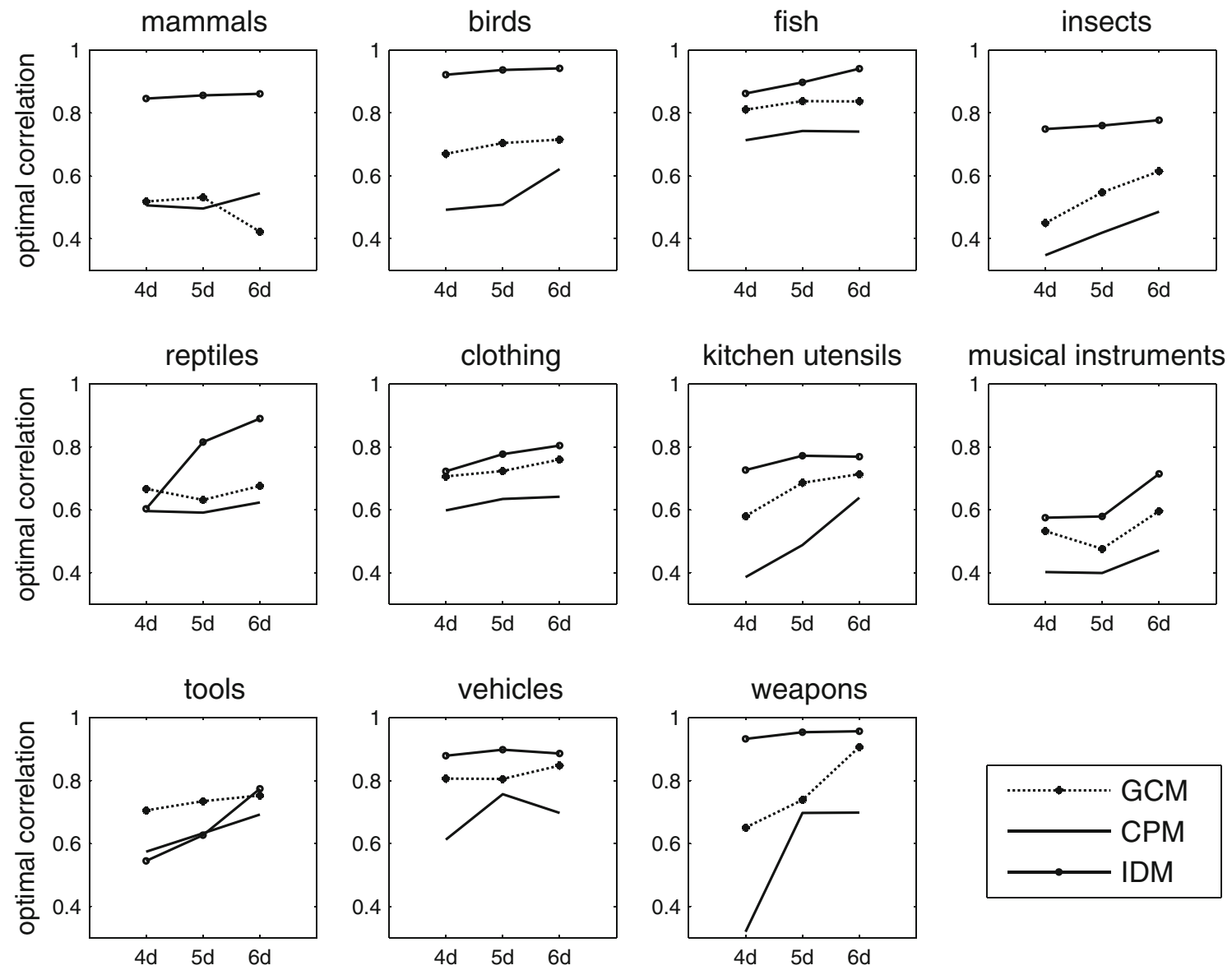

Fig. 3 Optimal performance of the IDM, GCM, and CPM in the prediction of typicality based on the underlying similarity space. The reported correlations are produced by optimal parameter settings

that the dimensional weights have to sum to 1 . For the sensitivity parameter, we used different priors: a uniform distribution and an approximation to the $\operatorname{gamma}(0,0)$ distribution (see Jaynes, 2003, Chap. 12; Lunn, Thomas, Best, \& Spiegelhalter, 2000). Upper boundaries were varied in between 5 and 50 . All results were qualitatively nearly identical, regardless of the exact choice of the prior. We report the results of the gamma prior, with upper bound set at 10, in Fig. 4.

Again, the results are largely in favor of the IDM. In particular, for 7 of the 11 categories, the IDM is clearly the better model. The GCM is the better model for only 2 out of 11 categories (clothing and reptiles). The CPM is not competitive. For the remaining 2 categories (kitchen utensils and vehicles), evidence is not in favor of either model consistently across dimensionalities.

In sum, the results of the model tests in terms of goodness of fit and generalizability support two conclusions. First, the IDM can adequately account for the empirically observed typicality gradient of the categories tested, providing good optimal fits of the typicality judgments. Second, for the majority of the categories tested in the present study, the IDM provides a better account of typicality than do the two traditional models of category representation.

\section{General discussion}

In classification of artificial stimuli and in the context of natural language categories, the importance of psychological extremes in representations has already been acknowledged. Earlier studies have highlighted psychological extremes in the form of extreme values relative to contrast categories (Davis \& Love, 2010; Goldstone, Steyvers, \& Rogosky, 2003; Palmeri \& Nosofsky, 2001), ideals originating from a logical rule (Nosofsky, 1991; Vandierendonck, 1988), the observation of a bias toward regular-but extremely rare - geometric figures (Feldman, 2000), goalderived categories (Barsalou, 1985), animal and artifact categories (Ameel \& Storms, 2006; Voorspoels, Vanpaemel, \& Storms, 2008b), and so forth.

In the present article, our aim was to formalize the notion of ideal representation - an instance of a psychologically 


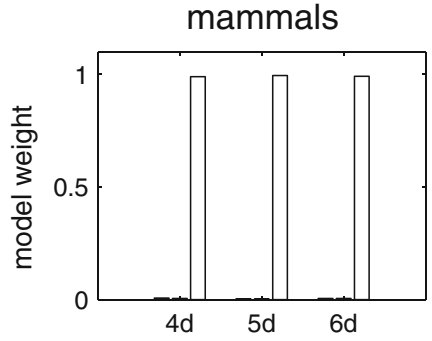

reptiles

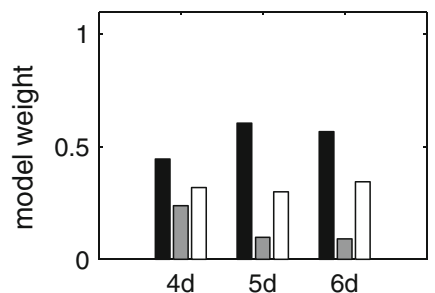

tools

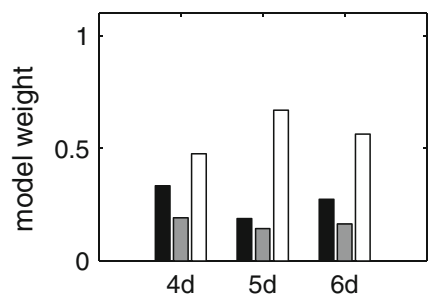

birds

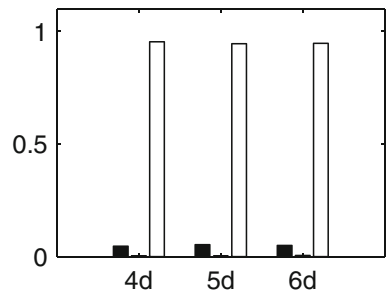

clothing

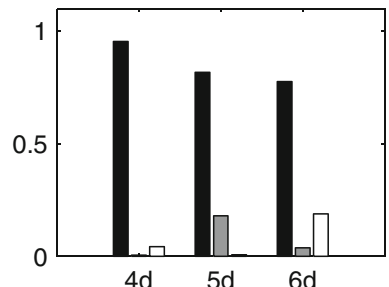

vehicles

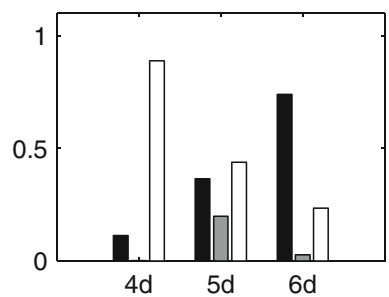

fish

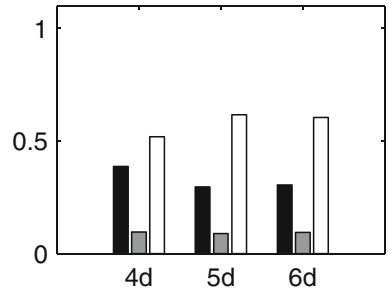

kitchen utensils

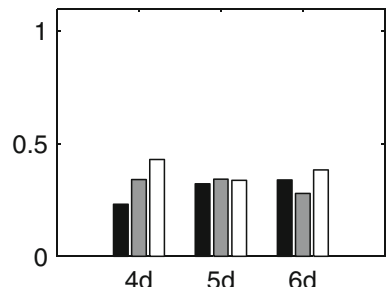

weapons

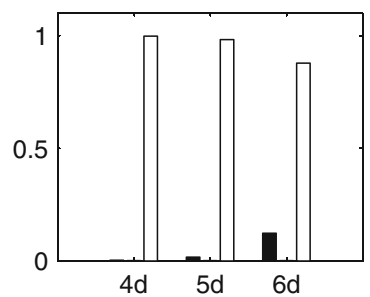

insects

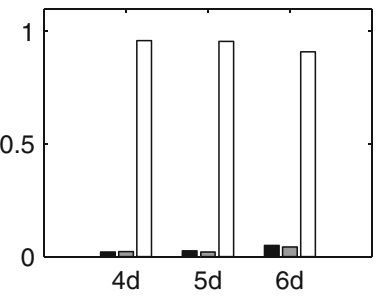

musical instruments
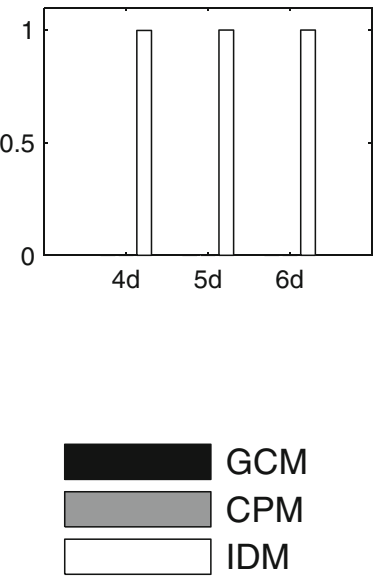

Fig. 4 Comparison of the IDM, GCM, and CPM in 11 natural language categories. Model weights reflect the relative evidence the data provide for each of the models

extreme representation - into a computational model, the IDM. The IDM passed the empirical test of accounting for the typicality gradient in superordinate natural language categories and was superior to the GCM and CPM in a majority of the categories tested. In what follows, we will discuss the interpretation of the IDM and the relevance of the model's success for the debate on abstraction in category representation.

\section{Interpretation of the ideal dimension}

We did not attempt a more substantial interpretation of the IDM for the specific categories in terms of what features constitute the ideal. Interpreting the combination of features that make up the ideal dimension was particularly difficult, since the axes of the spatial similarity representation were not interpreted. This, however, does not mean that the ideal dimension can never be verbalized. Two considerations are appropriate here. First, it is not structurally impossible to interpret the ideal dimension; rather, it is a consequence of the data used in the present study. If the IDM were to be applied using an a priori defined similarity space (e.g., one in which the axes refer to a specific feature), the ideal dimension can be thought of as a combination of the features that define the axes. In the present study, when no other information is available, the following, somewhat trivial interpretation can be made: A dog is typical for the category of mammals to the extent it has a lot of the combination of features that make up "mammalness." It is, however, not trivial that this combination of features, the ideal dimension, can be found in the category's similarity representation.

Second, the fact that the IDM does not require a substantial interpretation can be seen as a strength of the model. Interestingly, Barsalou (1985) considered ideals in common natural language concepts (as opposed to goal-derived categories). The ideals for the common categories were handpicked intuitively. For the category birds, the ideal dimension was how much people like it; for weapons, the ideal dimension was how effective it is. While for some of the categories (e.g., weapons) the ideal seems appropriate, this certainly was less obvious for other categories (e.g., birds). Lynch et al. (2000) have demonstrated that potential ideals for trees can be further away from everyday language (e.g., "weediness" for trees) for different types of tree experts. When there is no (explicit) goal involved in 
common taxonomic categories, the relevant ideal might even be further away from language.

The empirical success of the ideal approach should not depend on whether the ideal can be adequately verbalized. Internalized, implicit cultural norms regarding common taxonomic categories might motivate ideal dimensions that are not even easily recognized, let alone verbalized (Weiskopf, 2009). The IDM does not require verbalization of the ideal, thus offering the possibility of investigating ideal representations in a wider range of categories. This allows a larger scope of applicability of the IDM, for example, in common natural language categories where no intuitive goal or ideal dimension can be verbalized.

\section{Abstraction in category representation}

After more than 3 decades of research concerning artificial category learning and in research involving natural language concepts, it has been found across an impressively large array of conditions that exemplar models provide a better description of human categorization than do abstractionist prototype models. Nosofsky (1992) and Vanpaemel and Storms (2010) have provided overviews of this computational research. Nosofsky (1992) summarized 36 studies, of which only 6 favored a prototype representation over an exemplar representation. Vanpaemel and Storms (2010) reviewed 30 studies, all by Nosofsky and his collaborators, only 3 of which provided evidence for prototype representations. The rare studies comparing exemplar and prototype representations in natural language categories support the same conclusion: Exemplar representations are found to be superior to prototype representations (Voorspoels, Vanpaemel, \& Storms, 2008a). In short, there is little evidence for abstraction in category representation (but the debate is not yet settled; see Smith \& Minda, 2000).

The present study suggests that the traditional conception of a prototype as the average of a category may not be adequate but that abstraction, in some cases, can take different forms - for example, the form of ideal representations. Following this approach, we provided rare but compelling evidence for the existence of abstract summaries in category representation.

Interestingly, in this study, we focused on superordinate natural language categories, whereas most evidence supporting exemplar representations has been found in artificial categories of a more subordinate level. Therefore, these two factors, artificial categories versus natural language categories and the hierarchical level of the categories, deserve further exploration so that the present findings can be understood better and the debate on abstraction in category representation is further enriched.
Author Note Wouter Voorspoels, Wolf Vanpaemel and Gert Storms, Department of Psychology, K.U. Leuven.

The Research in this article is part of Research Project G.0281.06 sponsored by the Belgian National Science Foundation-Flanders, granted to the third author.

We want to thank Michael D. Lee for useful comments and suggestions.

Correspondence concerning this article should be addressed to Wouter Voorspoels, K.U. Leuven, Department of Psychology, ConCat, Tiensestraat 102, B-3000 Leuven, Belgium. Email: wouter.voorspoels@psy.kuleuven. be. Website: ppw.kuleuven.be/concat.

\section{References}

Ameel, E., \& Storms, G. (2006). From prototypes to caricatures: Geometrical models for concept typicality. Journal of Memory and Language, 55, 402-421.

Atran, S. (1999). Itzay Maya folkbiological taxonomy. In D. L. Medin \& S. Atran (Eds.), Folkbiology (pp. 119-204). Cambridge: MIT Press.

Barsalou, L. W. (1985). Ideals, central tendency, and frequency of instantiation as determinants of graded structure in categories. Journal of Experimental Psychology. Learning, Memory, and Cognition, 11, 629-654.

Brooks, L. R. (1978). Non-analytic concept formation and memory for instances. In E. Rosch \& B. B. Lloyd (Eds.), Cognition and categorization (pp. 169-211). Hillsdale: Erlbaum.

Burnett, R. C., Medin, D. L., Ross, N. O., \& Blok, S. (2005). Ideal is typical. Canadian Journal of Experimental Psychology, 59, 3-10.

Burnham, K., \& Anderson, D. (2002). Model selection and multimodel inference: A practical, information-theoretic approach. New York: Springer.

Carroll, J. D. (1972). Individual differences and multidimensional scaling. In R. N. Shepard, A. K. Romney, \& S. B. Nerlove (Eds.), Multidimensional scaling: Theory and applications in the behavioral sciences (Vol. 1, pp. 105-155). New York: Seminar Press.

Davis, T., \& Love, C. B. (2010). Memory for category information is idealized through contrast with competing options. Psychological Science, 21, 234-242.

De Deyne, S., Verheyen, S., Ameel, E., Vanpaemel, W., Dry, M., Voorspoels, W., et al. (2008). Exemplar by feature applicability matrices and other Dutch normative data for semantic concepts. Behavioral Research Methods, 40, 1030-1048.

Dry, M., \& Storms, G. (2009). Similar but not the same: A comparison of the utility of directly rated and feature-based similarity measures for generating spatial models of conceptual data. Behavior Research Methods, 41, 889-900.

Feldman, J. (2000). Bias toward regular form in mental shape spaces. Journal of Experimental Psychology. Human Perception and Performance, 26, 1-14.

Goldstone, R. L., Steyvers, M., \& Rogosky, B. J. (2003). Conceptual interrelatedness and caricatures. Memory \& Cognition, 31, 169-180.

Hampton, J. A. (1979). Polymorphous concepts in semantic memory. Journal of Verbal Learning and Verbal Behavior, 18, 441-461.

Heit, E., \& Barsalou, L. W. (1996). The instantiation principle in natural language categories. Memory, 4, 413-451.

Jaynes, E. T. (2003). Probability theory: The logic of science. Cambridge: Cambridge University Press.

Komatsu, L. K. (1992). Recent views of conceptual structure. Psychological Bulletin, 112, 500-526.

Kruskal, J. (1964). Nonmetric multidimensional scaling: A numerical method. Psychometrika, 29, 115-129. 
Lee, M. D. (2004). A Bayesian analysis of retention functions. Journal of Mathematical Psychology, 48, 310-321.

Lee, M. D., \& Wagenmakers, E. J. (2005). Bayesian statistical inference in psychology: Comment on Trafimow (2003). Psychological Review, 112, 662-668.

Lunn, D. J., Thomas, A., Best, N., \& Spiegelhalter, D. (2000). WinBUGS - a Bayesian modelling framework: Concepts, structure and extensibility. Statistics and Computing, 10, 325-337.

Lynch, E. B., Coley, J. D., \& Medin, D. L. (2000). Tall is typical: Central tendency, ideal dimensions, and graded category structure among tree experts and novices. Memory \& Cognition, 28, 4150.

Medin, D. L., \& Schaffer, M. M. (1978). Context theory of classification learning. Psychological Review, 85, 207-238.

Minda, J. P., \& Smith, J. D. (2010). Prototype models of categorization: Basic formulation, predictions, and limitations. In E. Pothos \& A. Wills (Eds.), Formal approaches in categorization (pp. 4064). Cambridge: Cambridge University Press.

Myung, I. J. (2000). The importance of complexity in model selection. Journal of Mathematical Psychololgy, 44, 190-204.

Myung, I. J., \& Pitt, M. A. (1997). Applying Occam's razor in modeling cognition: A Bayesian approach. Psychonomic Bulletin \& Review, 4, 79-95.

Nosofsky, R. N. (1986). Attention, similarity, and the identificationcategorization relationship. Journal of Experimental Psychology. General, 115, 39-57.

Nosofsky, R. N. (1988). Exemplar-based accounts of relations between classification, recognition, and typicality. Journal of Experimental Psychology. Learning, Memory, and Cognition, 14, 700-708.

Nosofsky, R. N. (1991). Typicality in logically defined categories: Exemplar-similarity versus rule instantiation. Memory \& Cognition, 19, 131-150.

Nosofsky, R. N. (1992). Exemplars, prototypes, and similarity rules. In A. F. Healy \& S. M. Kosslyn (Eds.), Essays in honor of William K. Estes: Vol. 1. From learning theory to connectionist theory;
Vol. 2. From learning processes to cognitive processes (pp. 149167). Hillsdale, NJ: Erlbaum.

Palmeri, T. J., \& Nosofsky, R. N. (2001). Central tendencies, extreme points, and prototype enhancement effects in ill-defined perceptual categories. Quarterly Journal of Experimental Psychology, 54A, 197-235.

Pitt, M. A., Kim, W., \& Myung, I. J. (2003). Flexibility versus generalizability in model selection. Psychonomic Bulletin \& Review, 10, 29-44.

Posner, M., \& Keele, S. (1968). On the genesis of abstract ideas. Journal of Experimental Psychology, 77, 353-363.

Rosch, E. (1975). Cognitive representation of semantic categories. Journal of Experimental Psychology. General, 104, 192-233.

Smith, J. D., \& Minda, J. P. (2000). Thirty categorization results in search of a model. Journal of Experimental Psychology. Learning, Memory, and Cognition, 26, 3-27.

Smits, T., Storms, G., Rosseel, Y., \& De Boeck, P. (2002). Fruits and vegetables categorized: An application of the generalized context model. Psychonomic Bulletin \& Review, 9, 836-844.

Vandierendonck, A. (1988). Typically gradient in well-defined artificial categories. Acta Psychologica, 69, 61-81.

Vanpaemel, W., \& Storms, G. (2010). Abstraction and model evaluation in category learning. Behavior Research Methods, 42, 421-437.

Voorspoels, W., Vanpaemel, W., \& Storms, G. (2008a). Exemplars and prototypes in natural language concepts: A typicality based evaluation. Psychonomic Bulletin \& Review, 15, 630-637.

Voorspoels, W., Vanpaemel, W., \& Storms, G. (2008b). Modeling typicality: Extending the prototype view. In B. C. Love, K. McRae, \& V. M. Sloutsky (Eds.), Proceedings of the 30th Annual Conference of the Cognitive Science Society (pp. 757-763). Austin TX: Cognitive Science Society.

Wagenmakers, E. J., \& Farrel, S. (2004). AIC model selection using Akaike weights. Psychonomic Bulletin \& Review, 11, 192-196.

Weiskopf, D. A. (2009). The plurality of concepts. Synthese, 169, $145-173$.

Younger, B. A., \& Cohen, L. B. (1983). Infant perception of correlations among attributes. Child Development, 54, 858-867. 